\title{
Leadership and scholarship in thoracic surgery
}

\section{Hartzell V. Schaff, MD}

Thank you for the kind introduction and welcome everyone to Minnesota. This is not the first meeting of the American Association for Thoracic Surgery (AATS) in our state. In 1924, the AATS held its sixth meeting in Rochester, the city where I live, and the home of the Mayo Clinic. Dr Carl Hedblom, who hosted the meeting, was the second of 7 thoracic surgeons associated with Mayo who have been president of the AATS. The other surgeons were Samuel Robinson, Stuart Harrington, Jim Claggett, and more recently Drs John Kirklin, Dwight McGoon, and Robert Wallace.

Minnesota has been an important focal point in the development of our specialty, and indeed, for one brief period beginning in 1955, Minneapolis and Rochester, Minnesota, were the only 2 cities in the world where open-heart surgery was being performed using extracorporeal circulation. This state continues to contribute to technology and biomedical development. There are more than 500 Food and Drug Administration-registered medical technology companies in Minnesota, and 280 of these are device companies. Indeed, the state is a leader in medical device research and manufacturing; per capita, Minnesota is number one, and in dollar terms, our state is second overall behind only California.

Serving this organization as a member at large, a Council member, and this past year as president has been a highlight of my professional career. Indeed, as a resident finishing training in 1980, I privately aspired to membership to 2 professional organizations. The first was the AATS, and the second was a surgical group that now is largely irrelevant to thoracic surgeons. As I looked ahead to a career in cardiothoracic surgery, I hoped to practice well, to teach well, and to contribute in some way to the knowledge base and science of our specialty. And to me, the sign that I had achieved these goals, at least partially, was membership to this Association.

From the Department of Cardiovascular Surgery, Mayo Clinic, Rochester, Minn. Disclosures: The author reported no conflicts of interest.

The Journal policy requires editors and reviewers to disclose conflicts of interest and to decline handling or reviewing manuscripts for which they may have a conflict of interest. The editors and reviewers of this article have no conflicts of interest.

Read at the 93rd Annual Meeting of The American Association for Thoracic Surgery, Minneapolis, Minnesota, May 4-8, 2013.

Received for publication Jan 26, 2021; revisions received Jan 26, 2021; accepted for publication Jan 27, 2021; available ahead of print Feb 16, 2021.

Address for reprints: Hartzell V. Schaff, MD, 200 First St SW, Rochester, MN 55905 (E-mail: schaff@mayo.edu).

J Thorac Cardiovasc Surg 2021;161:2138-44

$0022-5223 / \$ 36.00$

Copyright (c) 2021 by The American Association for Thoracic Surgery

https://doi.org/10.1016/j.jtcvs.2021.01.138

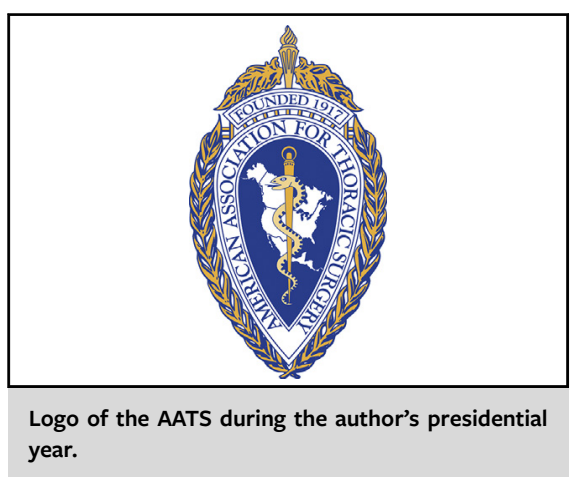

CENTRAL MESSAGE
Leadership and scholarship
define the AATS and its mem-
bers. Surgeons should recognize
the critical importance of role
models in surgery and consider
the unexpected benefits of
scholarly activity.

As most other Presidents have done, I want to acknowledge the many people who have helped me in my career, and my list may be longer than most because I have needed more assistance along the way. My parents were loving and supportive, both emotionally and financially, but they were firm in their belief that their children should be independent and well educated. I think my father would be especially proud of today. He spent his medical career in 2 small towns in Oklahoma and was a largely self-taught surgeon.

Today is special for me also because my family is here to share this honor, and to be able to meet many of you, my colleagues. Present today is my oldest child Brynn Hughes, and my second daughter, Leslie Kuckler with her husband Matt and 2 of our grandchildren Owen and Julia. Also here is my youngest daughter, Sarah, and my son Matt, who is a surgical intern at Mayo and is accompanied by his fiancée Caitlin Moore. And the most important person whose love and support have sustained me for the last 40 years is my wife Voni. Thank you all for being here today.

It is easy for me to trace my interest in thoracic surgery to the staff at the University of Oklahoma, where I was a medical student. Drs G. Rainey Williams, Lazar Greenfield, and 
Ron Elkins, and the residents who worked under them were, in my mind, the most knowledgeable, the most capable, and the most patient-focused staff in the whole Medical Center. I will say a bit more about Dr Williams later, but it was due to his encouragement and the support of Drs Elkins and Greenfield that I secured an internship and residency at Johns Hopkins Hospital. And during my 7 years in Baltimore, I had the great good fortune to train and learn under Dr Vincent Gott, Bob Brawley, and Tim Gardner our 82nd president.

An old cowboy once said, "Timing has a lot to do with the outcome of a rain dance." And for me, completing residency in 1980 was perfect timing because a position opened for a cardiac surgeon at Mayo Clinic. As a young staff member at Mayo, I learned more about cardiac surgery and patient care from Drs Dwight C. McGoon, Gordon K. Danielson, Francisco J. Puga, James R. Pluth, and from a very talented group of former and present colleagues in general thoracic surgery and in cardiovascular surgery. Each of these surgeons, the staff from Oklahoma, from Baltimore, and from Rochester has influenced me deeply but in different ways. But all were similar in that they had careers that were consequential; they were serious surgeons with purpose and dedication. They have taught hundreds of other young residents and staff, and I thank them for helping me come before you today.

Over the past year, some of you have asked casually about this presidential address wondering out loud what the theme might be and if it was difficult to come up with a topic. The truth is that the problem was not finding a subject but deciding on which subject. And, I would like to think that this reflects not a senior surgeon who has become opinionated, but the reflections of a surgeon who has been deeply engaged in our specialty in practice, education, and research, and cares about its future.

So I wish to address 2 areas that pertain directly to our Association, and those are leadership and scholarship. Because to me these 2 values, leadership, and scholarship, define the AATS and its members. Thankfully, for this address, I did not have to submit a long list of learning objectives, and you will be spared Continuing Medical Education questions. The simple goals of this presentation will be met if surgeons in the audience think a little differently about these 2 aspects of our professional life and learn more about 2 influential people you may not have known before. And I will warn you that I take some risk in this presentation, not because the material is particularly controversial, but because some of it may be misinterpreted, and I will explain.

\section{LEADERSHIP}

First leadership. On one level, thoracic surgeons, indeed all surgeons, must be leaders, leaders of your team in the operating room, and in the hospital at large. Surgeons are regularly called upon by medical colleagues for guidance in decision-making, in patient management, and most surgeons are involved at one time or another with administration and management of patient care areas, especially the operating room. Over the last 2 decades, emphasis on leadership has changed because of the financial pressures in medicine and the complexity of the academic medical environment that requires interaction with many others and prioritizes the team approach, integrated practices, and multidisciplinary committees. And let me emphasize that all of this is necessary because of the complexity of our society and medicine and our specialty; indeed, integration and teamwork have been the hallmarks of the Clinic in which I have worked for more than 30 years.

But I have been a student of leadership or at least a keen observer and would offer a few of what I consider important points for the younger members of our audience. There are dozens of leadership seminars available and advertised online, and there are a plethora of self-help books on leadership, an unusual number of which are displayed in bookstores in airports. And while some are interesting reads, most do not adequately distinguish leadership and management.

And here is the first risk that I take-I hope no one leaves this hall thinking that I don't understand or appreciate the value of good management as a characteristic of leadership. In our divisions and in our departments we have great management responsibilities, for fiscal accountability, quality metrics, and ensuring regulatory compliance. But in overemphasizing management, we risk overlooking other critically important characteristics of leadership. Indeed, preoccupation with process and administration has the unintended consequence of obscuring genuine leadership.

So, management skills aside, what makes a good leader? John Maxwell is an author of more than 60 books, most of them on leadership, and he has penned a long list of pithy quotes including one that many of you have heard, "People don't care how much you know until they know how much you care." And in one of his books, entitled The 5 Levels of Leadership, he describes the evolution of leaders; the first phase is recognition of the importance of evolving from authority to persuasion. ${ }^{1}$ In sizing up job opportunities as Division or Department heads in surgery, surgeon applicants need to nail down lines of authority and control, control over finances, clinical practice, personnel, and infrastructure. But realize that the authority that comes with the position, the rights you have, will not sustain you as a leader. Position is a poor substitute for influence; and using position makes you a boss, not a leader.

Inevitably, as a leader, you will have to make difficult decisions, and you will have those difficult conversations with staff. But if you find yourself too often falling back on authority to solve a problem, ask yourself why you need to do that, why you have not been able to persuade that person 
or persons of the correctness of your plan. Yes, you sometimes have to take an unpopular position, but the fact that you do not mind being unpopular has limited usefulness in leading, developing projects, and embracing change. Nothing is wrong with having a leadership position, but everything is wrong with always using position to get people to follow you.

So persuasion is important, but there are other qualities that I believe are essential to leadership, and these are present to a greater or lesser extent in all leaders whether in politics and government, team sports, business, or motorcycle gangs.

1. First, as a leader, you must live and adhere to the values of your group. This means that as a surgical leader you must reflect the values of surgeons. Avoid the trap of thinking that being chosen as a leader automatically gives you insights that your colleagues don't have. Try not to start a conversation with, "I am wearing my this hat or that hat." As a surgical leader, wear your surgical hat. Any other hats you wear in your organization should go on top of your surgical hat, not on your bare head.

2. Second, leaders should be expert in some domain of the group that you lead. This may not be vitally important in leading a large corporation or medical center, but for groups the size of clinical departments it is critical. That expertise may be in clinical practice, in research, or education, or all 3, but the point is that almost all successful leaders of surgical divisions or departments are recognized for achievement in some aspect of surgery. Leadership is persuasion, and you must be credible to persuade.

3. Third, the leader must have a vision and plan, and, most importantly, demonstrate the ability to accomplish goals for the organization. In short, you have to be effective in getting something done for your group, and this means an accomplishment for your group that your colleagues value. Another speaking invitation or visiting professorship may be personally rewarding, but this does little for your colleagues. Likewise, implementing an institutional directive to get operative notes signed more quickly may be necessary, but most colleagues will not consider this a product of your leadership. Advancing an agenda, getting something done for the group, is not something leaders do alone. In my experience, the best achievements are those that originate from the leader's vision but are taken up, modified, and implemented by your group. For the last 15 years, our cardiac surgical department has had yearly retreats where we outline our goals, both near-term and long-term, for practice, education, and research. And then we develop plans to accomplish the goals that involve each member. And most importantly, we review these during the year to benchmark our progress. Planning and execution through your group are important. I am often chagrined when I speak to a colleague and the conversation turns to the health of the practice, the number of cases, etc, and I hear that there is a great concern because of a loss of volume of cases to a competing surgical group or to cardiologists. I say chagrined because when I then ask about the group's plan for practice growth, 9 times out of 10 , I get a blank stare. Identifying a problem whether it is in practice, research, or education is not sufficient. The leader should articulate a plan shared among members and follow through.

4. Fourth, you need, as a leader, to have the interests of your colleagues over your own...you need to have their back, and not because leadership is a popularity contest. Indeed, your team's trust in you is most important when you are making an unpopular move. Your colleagues need to be persuaded because they know you watch out for them. They will go along with your decision even with misgivings if they trust that you consider their interests first.

5. Finally, the leader should be a role model. This seems almost too obvious, but it is important. And being a role model should be differentiated from being a mentor. The term mentor has always seemed inadequate to me. The concept of mentoring originated in Greek mythology. When Odysseus left for the Trojan War, he placed Mentor in charge of his palace and, more important, the care and upbringing of his son Telemachus. But mentoring often involves multiple individuals, and the word, mentor, is synonymous with counselor or guide. The term "role model," however, has a much more recent origin; it was coined in the first half of the twentieth century by Columbia sociologist Robert K. Merton and first appeared in the 1950s in the publication of a Columbia study of the socialization of medical students. ${ }^{2}$ Merton used the term role model when he hypothesized that individuals compare themselves with reference groups of people who occupy the professional and social roles to which the individual aspires. Indeed, we need role models, as thoracic surgery is recovering from a minor crisis in confidence because of a diminishing applicant pool. And there are many reasons for this, including changing demographics of medical schools, concerns over limited practice opportunities, and generational attitudes toward work. I won't discuss qualities of role models, but the point is that you have had many mentors but the role models were the surgeons who by dedication, skill, and character, drew you to our specialty. My first role model in thoracic surgery was Rainey Williams, whom I mentioned before, and I will share an encounter that I had with him that I have never forgotten. Although Dr Williams was later Chairman of Surgery, when I was in medical school, he was Chief of Cardiothoracic 
Surgery and a congenital heart surgeon. As a medical student on rotation in pediatric surgery, I was in the combined recovery room and intensive care unit at the Children's hospital writing postoperative orders and a note on an unfortunate burn patient who had undergone debridement. A child who had just had repair of tetralogy of Fallot was wheeled into the unit accompanied by the chief resident, the anesthesiologist, junior residents, and assorted nurses. Most of you recall the first time you watched with amazement at the coordination of the surgical team settling a patient in the intensive care unit. After a few minutes, Dr Williams entered, checked the monitor of his patient and the flurry of activity, and then walked over to where I was standing and asked what operation I had scrubbed on and what I had learned. We discussed the procedure and the plan for the patient, and then he asked how I enjoyed the rotation and encouraged me to consider an elective on thoracic surgery. He was impressive in many ways, and always respectful and interested in residents and students. I am sure that many of you are role models for your residents but consider this, at least 3 of Dr Williams's surgical residents named a son after him. Don't settle for being a mentor; be a role model.

\section{SCHOLARSHIP}

The second important aspect of our Association that I want to address is scholarship, the knowledge that results from study and research. There may be a stereotype where a busy clinician diminishes scholarship by saying that it is for someone else to do, and vice versa, an academic surgeon may feel that his or her grant is more important than looking after patients, because "the others can do that." It is my strong belief, however, that the best research is done by those who have clinical excellence because the clinician knows the important questions to ask and has insights into the best avenues for investigation. Likewise, the best clinicians not only have busy practices but have thought about and written about their experiences, so that others will use their methods and avoid the pitfalls they have observed.

I believe that scholarship in general, and research in particular, invigorates our specialty and is critically important if we expect to continue to attract the best and the brightest students to thoracic surgery. And this brings me to a second unintended consequence. The decline in applications to thoracic surgical residencies has been met with a host of potential solutions, some of which are necessary, and others that slightly miss the mark.

Take, for example, the issue of the length of training. Sixyear programs have great appeal, and most interpret this popularity as a desire of residents to shorten postgraduate training. That may be partly true, but I suspect that the more important reason is that students don't want to spend
5 years learning operations they will never perform as a cardiothoracic surgeon. So why then do we design 6-year programs with requirements for general surgical cases? Yes, basic surgical skills have to be learned along the way, but we in thoracic surgery should take ownership of that. And when does the thoracic surgical resident do research?

I have a simple test survey to submit to medical students who may be interested in thoracic surgery. Ask each applicant if he or she would rather have a 6-year thoracic residency containing 18 months of general surgery or a 6-year program with 6 months of general surgery and 1 year of research? My bet would be on the latter, and in fact, I strongly suspect that many students would be attracted to a 7-year thoracic program that included only 6 months of basic general surgery but had 2 years of research leading to a $\mathrm{PhD}$.

I believe that scholarship is important and that no one reads a medical publication the same way after writing a paper as they did before. Similarly, no one thinks about or writes a paper the same way after serving as a journal reviewer as they did before.

Research in thoracic surgery is very broad; it may involve molecular biology, integrated physiology, device development, or outcomes research. All of these domains are important to our specialty, but what are the benefits to the trainee? The first benefit to the resident might be something as simple and practical as career advancement. Some residents may choose to go into the laboratory to please their chief and increase the chance of securing a residency position or postresidency fellowship.

A second but important benefit of research is the satisfaction of discovery and recognition of the work through presentation and publication. Before a research experience, most residents have spent 5 to 7 years of medical school and residency absorbing as much of the whirlwind of information in medicine and science as possible. But residents are generalists, learning and retaining only the important information regarding patients and diseases. In contrast, during dedicated research time, the resident can study one problem in-depth and with focus can become a true expert in that area.

And third, in some instances, the laboratory experience will be a direct lead into a career in investigation as a surgeon/scientist. The fellow may be able to continue a line of investigation and even funding through later years of residency and into early career. This outcome might be considered the home run of resident research, but the fact is that this success occurs with a minority of residents.

But there is another benefit to the resident who has a research experience, the unexpected benefit that is rarely considered. Often, the techniques learned and the concepts mastered while doing research can be applied later to problems that are wholly unrelated to the original project. And I 
suspect that this happens much more than we appreciate. So let me finish with 2 examples of what I consider to be unexpected benefits of research, one from a scientist whom most of you do not know and the second, much more modest, from personal experience.

Earl Wood was born in Mankato, Minnesota, and received his medical degree and a doctorate in physiology from the University of Minnesota in 1941. He taught briefly at the University of Pennsylvania and Harvard before joining Mayo, where he remained until retirement in 1982. ${ }^{3}$ He had a long list of publications and patents, was a career investigator of the American Heart Association, and President of both the American Physiological Society and the Federation of American Societies of Experimental Biology. In the early 1970s, his yearly extramural funding was in the range of $\$ 1.5$ to $\$ 2$ million and his "purchasing power" was roughly the same throughout the 1960s. If you convert these numbers to 2013 dollars, this represents direct support of approximately 10 million dollars a year.

But what is relevant to us was his work in the Mayo Clinic Aero Medical Unit, which developed the first civilian human centrifuge that was used to test physiological responses to high levels of gravitational $(\mathrm{G})$ forces and led to the development of the G suit. ${ }^{4-6}$ Dr Wood often volunteered to ride in the centrifuge, which would increase $\mathrm{G}$ forces until the subject lost consciousness. ${ }^{7}$

Twenty-two different "M-style" G-suits developed at Mayo during World War II, and the M-21 entered the war on the North African front 1943 and was issued to all airmen by 1944 . A different G-suit from that same era was the prototype for flight suits used by pilots of the U-2 and SR-71 Blackbird. Dr Wood later served as a consultant to NASA on the Mercury project, which from 1961 through 1963 conducted the first 6 manned space flights for the United States.

But there were practical problems to overcome in these early studies of responses to gravitational forces. In the human centrifuge, the experimental subject was monitored by 2 observers, but they could not make hemodynamic recordings. Dr Wood and his team had to develop a variety of devices to measure physiological responses. You can't very well measure blood pressure in a spinning subject with a sphygmomanometer, so he modified an aircraft air pressure gauge into an instrument that became the standard tool for measuring arterial blood pressure. ${ }^{8,9}$ The ear oximeter was the first absolute-reading, self-calibrating unit ever devised, and was used in studies on the human centrifuge from 1942 to $1968 .^{10} \mathrm{He}$ and his team also developed a densitometer to measure indocyanine green dye curves and calculate cardiac output and, later, intracardiac shunts. ${ }^{11,12}$

In 1951, Dr Jeremy Swan came to Rochester to work with Dr Wood in the physiology research laboratory. He later joined the Mayo faculty as a cardiologist and was director of the catheterization laboratory working with Earl Wood. Together they made many important contributions in the study of circulation and intracardiac shunts, and he had a special interest in pulmonary hypertension in congenital heart disease. In 1965, Dr Swan moved to Cedars-Sinai Hospital in Los Angeles in 1965, and 5 years later, he and Willie Ganz developed the flow-directed pulmonary artery catheter. ${ }^{13}$

So the next time you settle a patient in the intensive care unit and you check the arterial blood pressure, oxygen saturation, and pulmonary artery pressures, and the cardiac output, recall that Dr Earl Wood played a major role directly or indirectly in the development of devices to measure these physiologic parameters. It is fair to say, however, that when developing these methods, Drs Wood and Swan had no inkling of the unexpected benefit of their research in managing cardiac surgical patients.

Unexpected benefits of research are not limited to devices and technology; sometimes concepts acquired during research can have later application in practice. During a research fellowship year, I worked in the laboratory of $\mathrm{Dr}$ Vincent Gott, another important role model for me in thoracic surgery. We and many others were investigating methods of myocardial protection, and the particular model that we used was a modified Langendorf preparation. The ex vivo environment was artificial, but it allowed tight control of many variables, and we could measure ventricular function reliably with an intraventricular balloon. The usual protocol involved stabilization of perfusion, an hour of global ischemic arrest, and 45 minutes of reperfusion. In one early study of myocardial protection, the best recovery of ventricular function after global ischemia function measured as isovolumic developed pressure or $\mathrm{dP} / \mathrm{dT}$ was seen in hearts that were cooled to $27^{\circ} \mathrm{C}$ and arrested with potassium cardioplegia. ${ }^{14}$ The most damaged hearts were those maintained at normothermia out cardioplegia.

However, an observation that was interesting to us was the increase in end-diastolic pressure that occurred when the intraventricular balloon was reinflated. After arrest, we introduced exactly the same volume into the balloon that before arrest resulted in an end-diastolic pressure of $10 \mathrm{~mm} \mathrm{Hg}$. In the damaged hearts after arrest and reperfusion, the end-diastolic pressure was elevated in almost direct proportion to the decrease in systolic function. So what was the mechanism for the elevated end-diastolic pressure?

We performed a simple set of experiments focusing on ventricular compliance. ${ }^{15}$ As expected, recovery of left ventricular systolic function was reduced in hearts maintained normothermia compared with those that had some degree of protection by hypothermia. In addition, end-diastolic pressure was greater in these hearts; further, there was increased myocardial water content in the hearts arrested 
normothermia with high-end diastolic pressure. We then constructed end-diastolic pressure-volume curves; the end-diastolic pressure-volume relationship is exponential so when the prearrest and postarrest curves are compared on a logarithmic scale, we saw no difference in the slope of the line but rather the postarrest curves was shifted upward and to the left. ${ }^{15}$ Thus, the pressure-volume curves suggest that the increase in end-diastolic pressure of postarrest hearts was due to a smaller unstressed volume rather than a change in compliance of the muscle. Said another way, the ventricular chamber size was smaller in hearts injured by ischemia/reperfusion, and refilling the ventricles to prearrest volumes led to increased pressure. In this isolated heart model, an important cause of the change in unstressed volume was edema, and in other studies, we used hyperosmolar perfusate during reperfusion to reduce water content and reduce end-diastolic pressure. ${ }^{15}$

So, in the research laboratory, I learned of the important relationship between ventricular volume and diastolic function. Now fast forward to the more recent era. At Mayo Clinic, we see and operate on a large number of patients with hypertrophic cardiomyopathy. Most patients referred for an operation will have subaortic obstruction and benefit from transaortic septal myectomy. However, a subgroup of patients with nonobstructive hypertrophic cardiomyopathy will be symptomatic with diastolic heart failure. And we encounter a number of symptomatic patients who have diastolic dysfunction and the particular anatomic subtype termed apical hypertrophic cardiomyopathy.

The apical form of hypertrophic cardiomyopathy is present in approximately $10 \%$ of patients in our hypertrophic cardiomyopathy (HCM) clinic but is much more common in Asia, where it accounts for approximately $25 \%$ of cases. We noticed that the most severely limited patients with apical HCM had what appeared to be very small ventricular chambers as a result of the apical muscle mass. Again, diastolic dysfunction can result from increased muscle stiffness which would be represented as a change in the slope of the pressure-volume curve, measured as the alpha constant in the exponential formula or as was true for the isolated hearts that had been injured by ischemic arrest and reperfusion in the experimental laboratory, elevated enddiastolic pressure in patients can be the result of a small left ventricular chamber size. Thus, the reduced compliance of the ventricle may not be due to a difference in muscle stiffness but smaller chamber size.

It seemed to us, then, that surgical enlargement of the ventricle by apical myectomy might improve diastolic function in symptomatic patients with apical HCM and small left ventricular chamber size. We enlarge the ventricle by extensive septal myectomy performed through an apical ventriculotomy, and the most important technical aspect is careful identification of the papillary muscles which are often displaced apically.
We remove muscle from the septum, the anterior wall of the ventricle, and occasionally shave the papillary muscles. The incision is closed in a fashion similar to that used for ventricular aneurysmectomy. First performed in September 1993 this operation has been used in more than 150 patients to relieve diastolic heart failure. ${ }^{16,17}$

How well does the procedure work? Postoperative hemodynamic studies have documented lower end-diastolic pressure following ventricular enlargement by apical myectomy. Of the first cohort of patients studied almost all had a reduction in left ventricular end-diastolic pressure, and in these same patients, there was a significant increase in ventricular end-diastolic volume. ${ }^{16}$ More detailed hemodynamic studies were performed as well. Individual left ventricular pressure-volume curves also demonstrated increased left ventricular stroke volume and decreased left ventricular end-diastolic pressure after apical myectomy for ventricular enlargement.

Would the operation for apical hypertrophic cardiomyopathy be developed without the previous laboratory study of diastolic function? That is hard to answer; I suspect the operation would be undertaken by someone at some time in the future, somewhere. However, I remember with crystal clarity the day the cardiologist who refers many patients with hypertrophic obstructive cardiomyopathy for surgery showed me the echocardiogram of a patient with apical disease and said, too bad we don't have a procedure for diastolic dysfunction...this patient's heart is too stiff. At that time I immediately recalled the diastolic dysfunction in reperfused hearts and thought that the problem in the patient might not be muscle stiffness per se, but reduced ventricular volume. And this extrapolation to an operation for diastolic dysfunction was an unexpected benefit of research, 20 years after my laboratory experience.

So I will close with my thanks for your patience in listening to this presentation. As I look out to you in the audience, I see present and future leaders in thoracic surgery. I hope you will take some time to think about the defining qualities of leadership and especially the importance of role models. And remember, too, of the unexpected benefits of scholarly activity. It has been a privilege and high, high honor to serve this past year as your president. Thank you.

\section{References}

1. Maxwell JC. The 5 Levels of Leadership: Proven Steps to Maximize Your Potential. New York: Center Street; 2011

2. Holton G, Robert K. Merton (4 July 1910-23 February 2003). Proc Am Philos Soc. $2004 ; 148: 505-7$.

3. Ritman EL. Earl Wood - a research career noted for development of novel instruments driven by the power of the indicator dilution concept. J Appl Physiol. 2014 117:945-56.

4. Wood EH. Special instrumentation problems encountered in physiological research concerning the heart and circulation in man. Science. 1950;112:707-15.

5. Wood EH. Evolution of instrumentation and techniques for the study of cardiovascular dynamics from the thirties to 1980, Alza lecture, April 10, 1978. Ann Biomed Eng. 1978;6:250-309. 
6. Wood EH. Prevention of the pathophysiologic effects of acceleration in humans: fundamentals and historic perspectives. IEEE Eng Med Biol Mag. 1991;10: 26-36.

7. Burchell HB. The investigator as volunteer subject. Mayo Clin Proc. 1982; 57( (suppl):28-33.

8. Lambert EH, Wood EH. Direct determination of man's blood pressure on the human centrifuge during positive acceleration. Fed Proc. 1946;5:59.

9. Lambert EH, Wood EH. The use of a resistance wire, strain gauge manometer to measure intraarterial pressure. Proc Soc Exp Biol Med. 1947;64:186-90.

10. Wood EH, Geraci JE, Groom DL. Photoelectric determination of blood oxygen saturation in man. Fed Proc. 1948;7:137.

11. Wood EH, Swan HJ, Marshall HW. Technic and diagnostic applications of dilution curves recorded simultaneously from the right side of the heart and from the arterial circulation. Proc Staff Meet Mayo Clin. 1958;33:536-53.

12. Swan HJC, Wood EH. Localization of cardiac defects by dye-dilution curves recorded after injection of T-1824 at multiple sites in the heart and great vessels during cardiac catheterization. Proc Staff Meet Mayo Clin. 1952;28:95-100.
13. Swan HJ, Ganz W, Forrester J, Marcus H, Diamond G, Chonette D. Catheterization of the heart in man with use of a flow-directed balloon-tipped catheter. $N$ Engl J Med. 1970;283:447-51.

14. Schaff HV, Dombroff R, Flaherty JT, Bulkley BH, Hutchins GM, Goldman RA, et al. Effect of potassium cardioplegia on myocardial ischemia and post arrest ventricular function. Circulation. 1978;58:240-9.

15. Schaff HV, Gott VL, Goldman RA, Frederiksen JW, Flaherty JT. Mechanism of elevated left ventricular end-diastolic pressure after ischemic arrest and reperfusion. Am J Physiol. 1981;240:H300-7.

16. Schaff HV, Brown ML, Dearani JA, Abel MD, Ommen SR, Sorajja P, et al. Apical myectomy: a new surgical technique for management of severely symptomatic patients with apical hypertrophic cardiomyopathy. J Thorac Cardiovasc Surg. 2010;139:634-40.

17. Nguyen A, Schaff HV, Nishimura RA, Geske JB, Dearani JA, King KS, et al. Apical myectomy for patients with hypertrophic cardiomyopathy and advanced heart failure. J Thorac Cardiovasc Surg. April 5, 2019 [Epub ahead of print]. 